\title{
Free-Form Rapid Prototyped Porous PDMS Scaffolds Incorporating Growth Factors Promote Chondrogenesis
}

\author{
Andrés Díaz Lantada, ${ }^{1}$ Hernán Alarcón Iniesta, ${ }^{2}$ \\ Beatriz Pareja Sánchez, ${ }^{1}$ and Josefa Predestinación García-Ruíz ${ }^{2}$ \\ ${ }^{1}$ Product Development Laboratory, Mechanical Engineering \& Manufacturing Department, \\ Universidad Politécnica de Madrid (UPM), c/José Gutiérrez Abascal 2, 28006 Madrid, Spain \\ ${ }^{2}$ Departamento de Biología Molecular, Universidad Autónoma de Madrid, 28049 Cantoblanco, Madrid, Spain
}

Correspondence should be addressed to Andrés Díaz Lantada; adiaz@etsii.upm.es

Received 7 October 2013; Accepted 29 November 2013; Published 2 January 2014

Academic Editor: Alexander Tsouknidas

Copyright (C) 2014 Andrés Díaz Lantada et al. This is an open access article distributed under the Creative Commons Attribution License, which permits unrestricted use, distribution, and reproduction in any medium, provided the original work is properly cited.

In this study, we present a promising approach for the rapid development of porous polydimethylsiloxane (PDMS) scaffold prototypes, with outer geometry defined from the design stage, according to the form of conventional implants or adapted to patients' biostructures. The manufacture method is based on phase separation processes using materials obtained by casting within additive rapid prototyped molds. We include a comparative study of PDMS sponges obtained by different simple processes. Final in vitro assessment is carried out using hMSCs (bone marrow-derived human mesenchymal stem cells), cultured onto porous PDMS scaffolds functionalized with aminopropyltriethoxysilane (APTS) and equilibrated with a trophic factors medium produced by the cells. Results show that porous PDMS scaffold prototypes are excellent 3D platforms for hMSCs adhesion. Furthermore, this PDMS3D niche, seeded with hMSCs and chondrogenic incubation medium during three weeks, showed a successful chondrogenesis determined by collagen type II expression. Thus, results show a versatile method to produce a 3D niche to address questions about cartilage and endochondral bone formation or skeleton tissues clinical approaches.

\section{Introduction}

Tissue engineering combines biological, physical, and engineering knowledge to provide artificially developed substitutes for tissues and organs linked to repair and replacement therapies. A key element involved in tissue engineering processes is the extracellular matrix or scaffold which serves as substrate or framework for cell growth, aggregation, and tissue development [1]. These scaffolds must be porous so as to allow cell migration during the colonization process as well as the transport of nutrients and waste to and from cells, and they have to be also resistant enough to withstand possible mechanical demands, especially if final scaffold (or device) implantation is desired. Additionally, as cells are able to feel their microenvironment and substrate texture upon which they lie by changing their morphology, cytoskeleton configuration, and intra- and extracellular signaling, increasing efforts are continuously being focused on advanced design and manufacturing technologies, so as to generate and modify the structures and surfaces of biomaterials. Aspects such as porosity, pore size, and surface microtexture promote cell adherence, migration, and proliferation within the scaffold, for subsequent differentiation into relevant cell types. Thus, tissue progenitor cells and the scaffold play a fundamental role in most tissue engineering strategies as their properties can deeply influence the global success of new tissue formation and the controlled fabrication of the scaffold structures is becoming increasingly important for novel approaches within regenerative medicine [2-4].

Most processes for manufacturing microporous structures for tissue engineering [5] involve combination of materials in some step of the process and a final phase separation, for obtaining a solid part with distributed small pores. Among most extended processes, gas-assisted injection molding is an industrial method based on injecting a molten resin or thermoplastic into a mold cavity and then applying a quantity 
of pressurized gas into the resin, so as to help to fill out the mold cavity and to create hollows and pores in the polymer. The incorporation of foaming agents as additives to polymers also allows the manufacture of polymeric parts with pores. In many strategies including tissue strategies, to obtain $3 \mathrm{D}$ porous structures is absolutely required to irrigate the tissue and maintain an adequate liquid dynamics. The use of porogens is also commonplace; normally, the process involves mixing a liquid prepolymer with solid particles (typically wax, sugar, salt, etc.). Once polymerization is produced, normally by UV exposure or by heating, a solid structure, formed by a polymeric network with dispersed particles, is obtained. Final porous structure is obtained by dissolving such disperse particles in water or other solvents or by heating. The use of prepolymer-water emulsions is also typical for obtaining a polymerizable mixture that after thermal or UV-based polymerization provides a polymeric network with pores according to initial water content (i.e., polyHIPEs).

Main alternative, for improving the control of scaffolds' pore size and distribution, from the design stage, is the use of micro additive manufacturing technologies (AMT), normally working on layer-by-layer processes, following the geometries obtained with the help of computer-aided designs $[6,7]$. Electrospinning can be also adapted to "layer-by-layer" fabrication and used for obtaining $3 \mathrm{D}$ porous structures [8], even though the process is not as repetitive as the use of micro AMT. The progressive increase in precision of additive manufacturing technologies, together with their improved versatility, thanks to a continuously increasing set of materials available for layer-by-layer processing, is greatly promoting applications linked to micro- and even nanomanufacturing of complex 3D geometries for very innovative medical solutions in several fields [5].

Several scaffolds with controlled microstructures have been manufactured using different RP technologies, such as selective laser sintering [9], layered hydrospinning [10], laser stereolithography [11], digital light projection [12] or twophoton lithography [13], and different materials including hydrogels [14], gelatin [7], titanium alloys [15, 16], some photopolymers [17], and ceramics [18]. However, in vitro validation of such rapid prototyped scaffolds is not so common, as most processes/materials do not provide an adequate combination for promoting biocompatibility and in many cases generate toxic components. Nevertheless, some highly interesting research has already been published, including in vitro validation and toxicity assessment $[19,20]$. Advances in the field of biomedical polymers [19-21] together with the possibilities of chemical/physical-vapor deposited (CVDPVD) coatings for biocompatibility enhancement [11] are bringing new possibilities to this area, although access to such materials and technologies is not always easy, as some of them are currently under development.

In any case, it seems clear that a universal methodology for tissue engineering scaffold development is not yet available, first of all due to the complexity of biological materials and systems and also due to all the possible design resources, manufacturing technologies, and related materials available, whose results have not been systematically compared. For instance, additive manufacturing technologies allow precise control of final geometries from the design stage; however, such designs are normally obtained by combining Euclidean based (simple) geometries and final result does not mimic adequately the complexity of biomaterials. On the other hand, scaffolds obtained by phase separation and more "traditional" processes typically lead to more biomimetic sponges, even though their final outer form and repeatability are more difficult to control than using computer-aided strategies linked to rapid prototyping using additive processes.

Therefore, further research is needed to address the advantages of combining different technologies [22] for manufacturing enhanced, even personalized, scaffolds for tissue engineering studies and extracellular matrices with global (outer) geometries defined as implants for tissue repair. In addition, increasing data show that progenitor cell-niche formation is absolutely needed for tissue development and repair [23]. Indeed, the niche composition and 3D structure play an important role in stem cells state and fate. The niche is created by the specific combination of trophic factors produced by progenitor cells to maintain capability for tissue repair and regeneration and specific extracellular matrix. Recent studies have helped to highlight the extreme relevance of the incorporation of adequate growth factors, within the scaffold, for promoting biological regulation, cell differentiation, angiogenesis, and final tissue viability [24-26].

The term "adequate growth factors" makes reference to a chondrogenic medium that was proposed at the laboratory of Arnold I. Caplan who coined the term mesenchymal stem cell, as described in Materials section. Such inclusion of biochemical effects, derived from the incorporation of the adequate growth factors, adds some additional uncertainties to the already complex to understand interactions between scaffolds' structure, morphology, and mechanical properties. Consequently, studies addressing these interesting synergies between extracellular matrices and growth factors and their impact on final tissue viability are needed, in the quest for the aforementioned universal methodology for versatile and successful tissue engineering scaffold development.

In this study we show an approach for the rapid development of porous polydimethylsiloxane (PDMS-3D) scaffold prototypes, with versatile outer geometry defined from the design stage, according to different tissue strategies, to the form of conventional implants or even adapted to patients' biostructures. The manufacture method is based on phase separation processes using parts obtained by casting within additive rapid prototyped molds. We include a comparative study of PDMS sponges obtained by different simple processes, including sugar leaching after polymerization, water extraction after polymerization of PDMS-water emulsions, and water extraction together with sugar leaching after polymerization of PDMS-sugar-water mixtures. By changing the proportions of these agents, main mechanical properties of the final scaffold can be tuned and adapted to the typical density and stiffness of several soft tissues. Different porous annuloplasty rings are also obtained, by means of example, so as to show the potential of these manufacture processes, towards free-form porous implants and scaffolds for tissue replacement and repair. 
Results show that PDMS scaffolds functionalized with trophic factors produced by hMSCs isolated from bone marrow became a PDMS-3D niche for cells adhesion and chondrogenesis. Furthermore, the PDMS functionalized with APTS became an excellent niche for hMSCs for chondrogenesis that was increased if the PDMS-APTS structures were treated with trophic factors before being seeded with hMSCs. Thanks to the described process, scaffolds became buried of collagen type II, even within the porous structure of the material. Thus, we recommend these scaffolds for cartilage and endochondral bone formation/repair strategies.

\section{Materials and Methods}

2.1. Materials. Microporous PDMS has already been proposed as a functional material for cell culture where high degree of porosity is required to improve cell survival and functions. PDMS sponges have been obtained using precursor microemulsions $[27,28]$ and other processes based on particle (usually salt or sugar) leaching [29]. Mixtures of PMDS-sugar-water, for promoting multiscale porosities, are less common. In addition, porogen size and content are normally used as main control parameters for adjustment of final mechanical properties, although assessment of such mechanical properties is not so common $[30,31]$. In this study we try to provide additional details about these special mixtures, considering also the proportions of the different agents and their impact on density, stiffness, and tensile strength, for additional comparison with soft tissues for potential replacement of repair.

For obtaining the PDMS sponges, we use a commercial Neukasil RTV-20 prepolymer, together with the Neukasil A2 cross-linker, both of them from Altropol Kunststoff GmbH (Rudolf-Diesel-Strasse, 9-13, D-23617, Stockelsdorf, Germany). Both components are mixed according to manufacturer's data sheets and introduced in a vacuum chamber, at the beginning of polymerization, for adequate degasification. Complete polymerization at room temperature $\left(25^{\circ} \mathrm{C}\right)$ lasts around 24 hours, which helps to manipulate the mixture with enough time for adequate molding. As porogen material we use Native Organic sugar (Balbo Group, Brazil), mixed in different proportions with PDMS and with PDMS-water emulsions. The mixtures analyzed in present study include the following weight distributions: $50: 50 \mathrm{PDMS} /$ water, $33.3: 33.3: 33.3$ PDMS/water/sugar, 50 : $50 \mathrm{PDMS} /$ sugar, $40: 60 \mathrm{PDMS} /$ sugar, 33.3:66.6 PDMS/sugar, and 28.5:71.5 PDMS/sugar, which lead to typical porosities used in tissue engineering and studies linked to scaffold development. A summary of the different compositions and morphologies studied and obtained, after particle leaching and drying (see process description below), is included in Figure 3, as brief summary.

Epoxy resin Accura 60 (3D Systems, 333 Three D Systems Circle, Rock Hill, SC, USA) is used as a base material for the rapid molds, subsequently used for casting the PDMS-sugarwater mixtures and for obtaining outer geometries controlled from the design stage, due to restrictions imposed by the laser

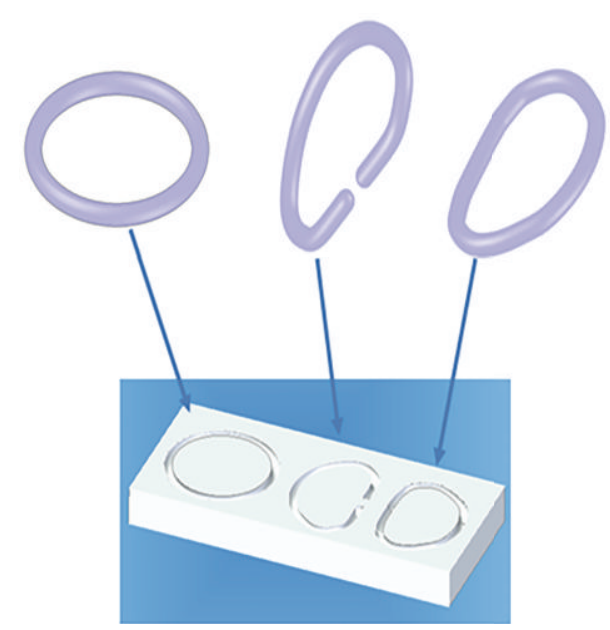

FIGURE 1: Computer-aided designs of different valve ring models and associated mold geometry.

stereolithography prototyping process used for such molds, as detailed below.

2.2. Design and Prototyping Processes. Several state-of-the-art implants aimed at tissue repair have circular or quasicircular ring geometries, from artificial sphincters to annuloplasty rings for heart valve (usually mitral) tissue reinforcement. These implants can greatly benefit from having controlled porosity and mechanical properties, as the pores can help to promote drug-eluting approaches for minimizing postsurgical risks, as well as allow for scaffold-based tissue engineering strategies. For instance, recent research has highlighted the potentials of rapid prototyped porous scaffolds for cardiac tissue engineering [32] and their adaptation to different cardiac prostheses, such as reinforcement valve rings; even using personalized designs [33] is almost direct.

As cases of study for present work, we have selected a circular ring, as possible reinforcement for tissues surrounding different sphincters, and a couple of mitral valve annuloplasty rings (one closed and one open) for potential reinforcement of valve tissue, as repair treatment for mitral valve insufficiency. These designs are aimed at verifying the possibility of obtaining PDMS sponges, with controlled outer geometries, by casting into rapid prototyped molds of different emulsions and mixtures including PDMS, sugar, and water.

The different implantable rings and associated mold geometries, as shown in Figure 1, are designed with the help of the computer-aided design software Solid Edge v.20 (Siemens Product Lifecycle Management Software Inc.) and are saved as stl files for subsequent communication with prototyping machines. The stl files with the $3 \mathrm{D}$ virtual CAD geometry of the mold designs are transferred to a laser stereolithography SLA-3500 machine (3D Systems, 333 Three D Systems Circle, Rock Hill, SC, USA) for subsequent rapid mold manufacture and casting of the PDMS mixtures (see also Figure 4). The process is based on an additive layerby-layer photopolymerization of an epoxy resin that ends 


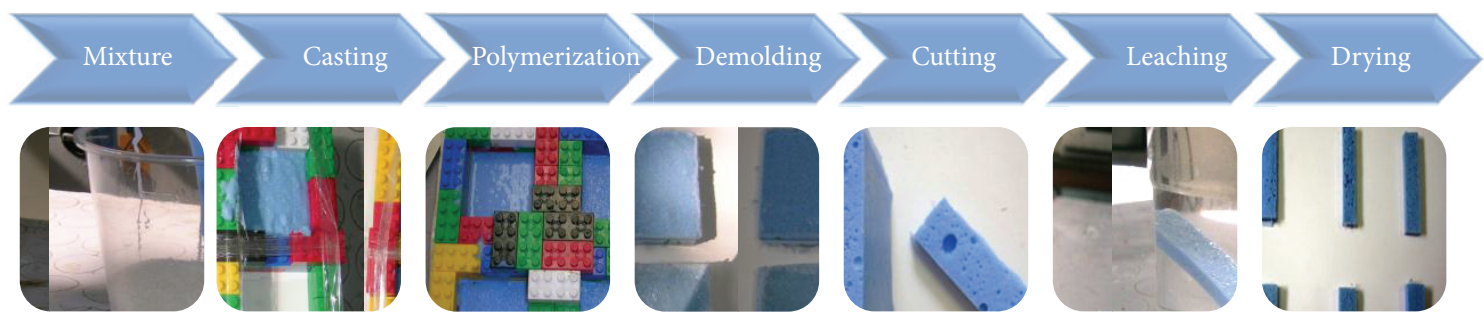

FIGURE 2: Overview of the manufacturing process used for obtaining PDMS sponges.
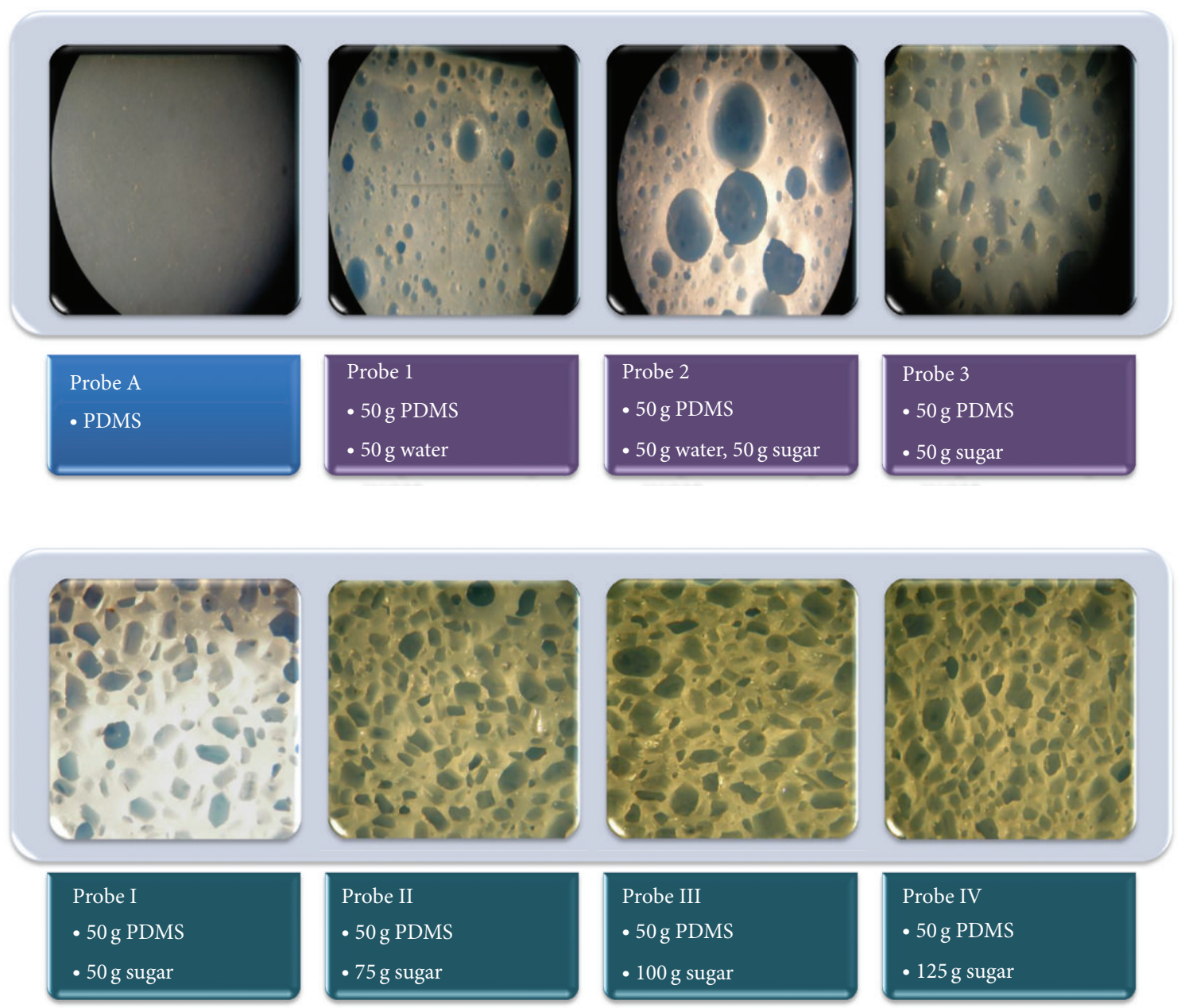

FIGURE 3: Different morphologies, after particle leaching and drying, obtained by systematic modification of the sugar and water content incorporated to the PDMS during the polymerization process.

up with a whole physical three-dimensional part. The SLA3500 system available at the UPM Product Development Laboratory allows us to manufacture details down to about $150 \mu \mathrm{m}$, although more recent studies on the development of microstereolithography are gradually increasing its precision to submicrometric scales (NanoScribe $\mathrm{GmbH}$ ), in spite of greatly limiting final part size to only a few $\mathrm{mm}^{3}$.

The prototyping process, either for the manufacture of porous PDMS scaffold probes or for the development of porous PDMS implants, includes several simple steps detailed further on and shown schematically in Figure 2. First of all, the Neukasil RTV-20 prepolymer and the Neukasil A2 cross-linker are mixed and degasified, following manufacturer's instructions, as previously explained. Subsequently, the PDMS-water emulsion or the PDMS-sugar or PDMSwater-sugar mixtures are obtained with the help of a Taurus vertical mixer-blender spinning at 1200 r.p.m. during one minute just before casting.

Both open and closed molds can be used, as well as atmospheric and vacuum casting, depending on the desired final level of detail (vacuum casting helps to fill the molds and to promote replication quality). In our case, such mixtures 

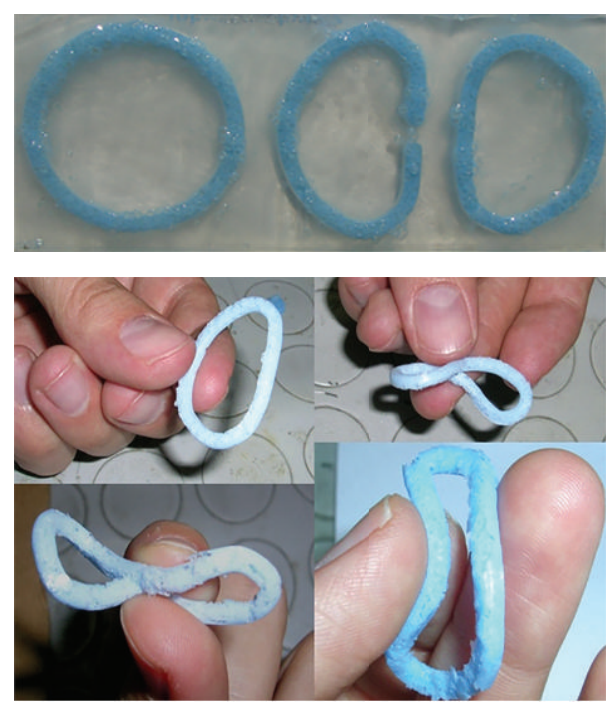

FIGURE 4: Example of the obtained porous PDMS annuloplasty rings aimed at valve tissue repair. Image from the PDMS rings casted into the rapid epoxy mold and details from the final parts.

are casted into rapid LEGO molds and into rapid prototyped epoxy molds for polymerization during 24 hours at room temperature. Once the PDMS mixtures are polymerized, demolding is accomplished for obtaining the desired implants or probes for mechanical testing, after adequate cutting. Particle leaching is achieved by water immersion and systematic squeezing of the material, probes, and prototypes. Final drying leads to the desired probes (see Figure 3) and implants (see Figure 4).

\subsection{Mechanical Characterization. Mechanical characteriza-} tion is addressed by tensile testing using a MTS 835 Damper Test System (MTS, 14000 Technology Drive, Eden Prairie, MN, USA), capable of applying compressive, tensile, and fatigue cycles (the machine and experimental setup are shown in Figure 5). In our case, the different $48 \times 10 \times 5 \mathrm{~mm}^{3}$ porous PDMS probes obtained are submitted to tensile testing until breakdown (see detailed view from Figure 5) and the different stress-strain curves, resulting from such traction tests, are represented in Figure 6 for further discussion. The initial probe length between clamps is $30 \mathrm{~mm}$ and the tensile tests are carried out at a speed of $10 \mathrm{~mm} /$ minute.

The influence of material processing and of the different proportions of components, on the final physical properties of the PDMS sponges, are detailed in the following section, together with a comparative analysis of the mechanical properties of common soft tissues.

2.4. Cell Culture Processes and Imaging Techniques. The specific composition of the adequate chondrogenic growth factors, in comparison with other osteogenic or adipogenic ones, is reported by Pittenger et al. [34] and used in some references also aimed at chondrogenic differentiation [3537]. In our case, two to four milliliters of human bone marrow samples from healthy donors were provided by Dr. Benjamin
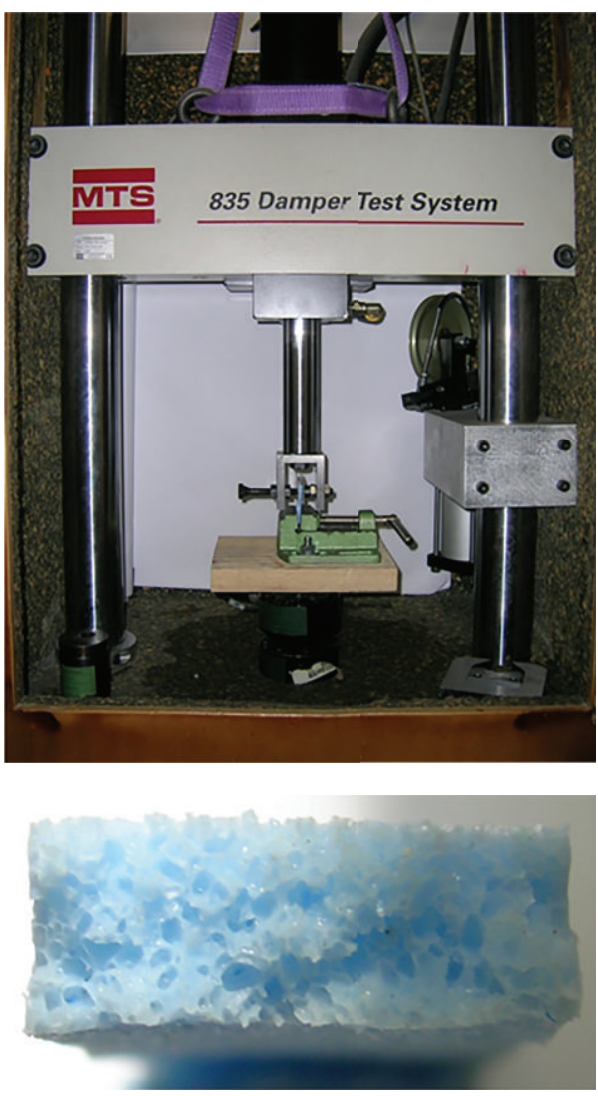

FIgURE 5: MTS 835 Damper Test System and result from a traction test carried out with porous PDMS.

Fernández from the Fundación Jiménez-Diaz. The culture expansion of hMSCs was carried out, as previously described $[35,36]$.

Cells were plated and incubated using DMEM-L plus $10 \%$ FBS of selected batches. Cells were collected by treatment with $0.25 \%$ trypsin-EDTA. Cell culture media were prepared by the research services of Molecular Biology Center "Severo Ochoa" (CSIC-UAM). For the preparation of hMSCs trophic factors rich medium we used 8-10 p100 culture plates at $80 \%$ confluence for each batch. Cells were washed with PBS and starved of FBS during 24 hours. Afterwards, culture medium was collected and cleaned of any floating cell by centrifugation at $1.500 \mathrm{rpm}$ in a bench centrifuge during $5 \mathrm{~min}$. The clean supernatant was cooled down on ice during $30 \mathrm{~min}$, centrifuged in a Sorvall to remove salt precipitations, and kept in $2 \mathrm{~mL}$ aliquots at $-30^{\circ} \mathrm{C}$ until use. We avoid any samples with repeated freeze-thaw.

Cubic pieces of porous PDMS scaffolds were UV irradiated, individually placed in $25 \mathrm{~mL}$ Falcon tubes, and received the following treatment: (i) thoroughly wash using $0.5 \mathrm{~mL}$ PBS and 5 min centrifugation until porous PDMS float stops; (ii) treatment with $2 \mathrm{M}$ acetic acid during $20 \mathrm{~min}$ and then a rapid neutralization and PBS wash; (iii) treatment with hMSC trophic factors medium during 24 hours or DMEMLG as control; and (iv) seed scaffolds with 150.000 hMSCs in $0.5 \mathrm{~mL}$ chondrogenic or control medium during three weeks 


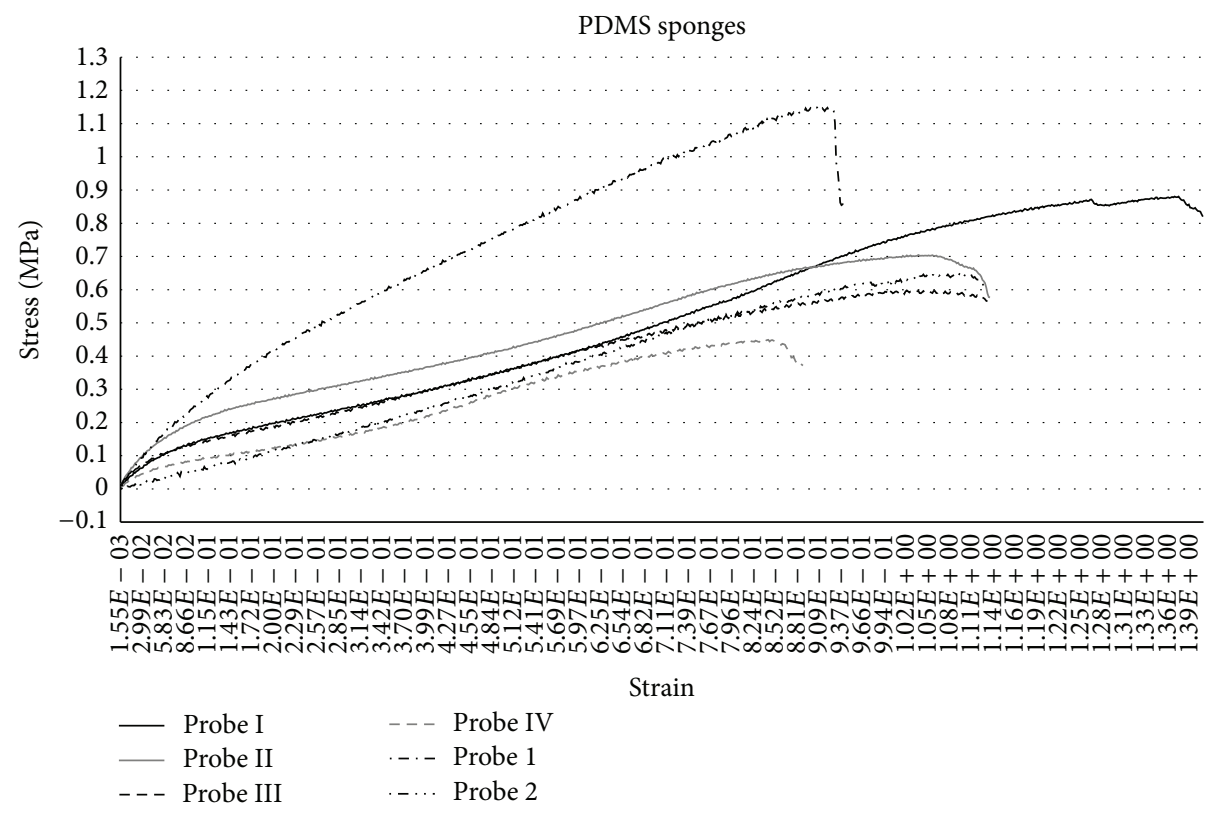

FIGURE 6: Comparative mechanical performance of the PDMS sponges obtained.

at $37^{\circ} \mathrm{C}$ in $5 \% \mathrm{CO}_{2}$ as early indicated [34-37]. When PDMS sponges were functionalized with APTS it was used at a concentration of $0.025 \%$ in ethanol. The chondrogenic medium was DMEM-LG with ITS $(6.25 \mu \mathrm{g} / \mathrm{mL}$ insulin, $6.25 \mu \mathrm{g} / \mathrm{mL}$ transferring, and $6.25 \mu \mathrm{g} / \mathrm{mL}$ selenous acid) (Collaborative Research), $1 \mathrm{mM}$ pyruvate (Gibco), $37.5 \mu \mathrm{g} / \mathrm{mL}$ ascorbate (WACO), $0.1 \mu \mathrm{M}$ dexamethasone (Decadran, Merck), and 0.06 ng TGF- $\beta$ (R\&D System).

Then, samples were processed to analyze by using an immune fluorescence technique, as described previously [37, 38], with some modifications. Briefly, cubic scaffolds were individually placed in M24 tissue dishes, cut in slices, rinsed with ice-cold PBS, fixed in 3.7\% formaldehyde in PBS during $30 \mathrm{~min}$ at RT, and washed in PBS. Cells were visualized by incubation with $0.5 \%$ Triton in CSK buffer containing $10 \mathrm{mM}$ pipes, $\mathrm{pH} 6.8,3 \mathrm{mM} \mathrm{MgCl}_{2}, 100 \mathrm{mM} \mathrm{NaCl}, 1 \mathrm{mM}$ EGTA, $0.3 \mathrm{M}$ sucrose, and $1 \mathrm{mM}$ PMSF for $30 \mathrm{~min}$ on ice. After the treatment, samples were cleaned and fixed with $3.7 \%$ formaldehyde and equilibrated in PBS. For collagen type II immune detection, scaffolds preparations were blocked of unspecific antibodies binding by incubation during an hour with $3 \%$ BSA-0.1\% Triton X-100 in PBS (PBSA) at RT or overnight at $4^{\circ} \mathrm{C}$. As primary antibodies anti-collagen type II at 1:200 were used, secondary antibodies were Alexa594conjugated from Molecular Probes, Eugene, OR, at 1/500 dilution. Nuclei were stained with DAPI (CALBIOCHEM). Immune stainings of the cell preparations were determined with an inverted IX81 Olympus with a DP72 digital camera.

\section{Results and Discussion}

Results from Figure 4, showing implant geometries with scaffolding porous structures, have helped to validate the design and rapid manufacturing processes, as relevant tasks towards a systematic procedure for the development of freeform, porous, and biomimetic scaffolds (as well as other (micro) implants) for tissue engineering and repair. The term "biomimetic" in this case makes not only reference to the appearance, but also reference to the mechanical properties, which are discussed below. The lower image of Figure 5, after probe collapse during tensile testing, helps to show the three-dimensional interconnected microporous network and the efficiency of the leaching process, as all the sugar particles have disappeared. Figure 6 includes the comparative mechanical performance of the PDMS sponges obtained and the most interesting values (tensile strength and Young modulus), together with density, are summarized in Table 1, besides interesting data about different typical soft tissues for additional reflections.

According to initial expectations, an increase of porogen loading (Probes I-IV) leads to lower scaffold densities and to lower values of tensile strength and Young modulus. The PDMS-water emulsion (Probe 1) leads to a density and Young modulus similar to those of Probe IV, while its tensile strength is quite higher. We explain such difference of tensile strength due to the smaller pore sizes and more homogeneous pore distribution of Probe 1, which helps to minimize the effects of stress concentration at the connections between pores.

However, pore sizes obtained by phase separation, after polymerization of the PDMS-water emulsion, are around 30 to 60 microns in diameter, smaller than the 100-200 microns in diameter pores obtained by particle leaching. The smaller pore sizes, in our case, prevent the formation of a three-dimensional network of interconnected pores, which is necessary for adequate scaffolds. Such interconnected pore network is obtained in Probes I-IV, with remarkable results in Probe IV, which we find the most apt for final in vitro validation via cell culture. Probe 2 , obtained by a combined 
TABLE 1: Results' summary from the mechanical characterization tests of the PDMS sponges obtained and comparative data from other porous polymers and natural soft tissues.

\begin{tabular}{lccc}
\hline $\begin{array}{l}\text { Porous PDMS } \\
\text { and tissues }\end{array}$ & $\begin{array}{c}\text { Tensile } \\
\text { strength } \\
(\mathrm{MPa})\end{array}$ & $\begin{array}{c}\text { Young } \\
\text { modulus } \\
(\mathrm{MPa})\end{array}$ & $\begin{array}{c}\text { Density } \\
\left(\mathrm{g} / \mathrm{cm}^{3}\right)\end{array}$ \\
\hline Probe I & 0.88 & 2.77 & 0.96 \\
Probe II & 0.71 & 3.70 & 0.82 \\
Probe III & 0.59 & 2.47 & 0.79 \\
Probe IV & 0.45 & 1.47 & 0.65 \\
Probe 1 & 1.15 & 1.64 & 0.64 \\
Probe 2 & 0.65 & 0.72 & 0.75 \\
PolyHIPE [39] & 0.17 & 0.88 & 0.15 \\
Skin [40] & 10 & 40 & 1.09 \\
Pericardium [41] & 25.8 & 156 & 1.8 \\
Tendon [40] & 80 & 1000 & 1.2 \\
Carotid artery & 0.8 & 0.97 & 1.3 \\
[40] & & 6.5 & 1.39 \\
Aorta artery [40] & 0.9 & 6 & 1.18 \\
Esophagus [42] & 0.5 & 5 & 0.87 \\
Parenchyma [43] & 0.6 & $0.45-0.9$ & $1.05-1.2$ \\
Cartilage [44] & $10-30$ & &
\end{tabular}

approach using a PDMS-sugar-water mixture, can be used for multiscale approaches, as different pore sizes are obtained, although some pores reaching diameters of 300-400 microns lead to a much lower Young modulus.

In any case, it is interesting to highlight the possibility of adjusting scaffold density, stiffness, and strength, almost ad hoc, by incorporating different quantities and mixtures of water, sugar, and water-sugar, to the PDMS. Previous research has verified the effect of scaffold stiffness on hMSCs differentiation and final fate, showing that softer scaffolds lead to softer tissues and that stiffer scaffolds lead to harder tissues. In our study, the scaffolds developed show a wide range of Young moduli, which can adapt to the requirements of several soft tissues, from cartilage to esophagus, parenchyma, and arteries, as shown in Table 1.

Regarding in vitro assessment via cell culture, results after three weeks (summarized in Figure 7) show that cells are well attached to the scaffold, even having penetrated into the porous structure, forming a three-dimensional network within the interconnected pores. For the cell culture process we have used Probe IV, as usually scaffold manufacturing processes for soft tissue repair pursue low densities and high porosities. The figure shows details of the cells attached to the scaffold surrounding and filling different pores. It is also relevant to note that, during cell culture, cells developed collagen, helping to show the potential for tissue repair of the proposed approach.

Scaffold's porosity, through capillary action, helps the growth factors to fill the three-dimensional interconnected porous structure, which proves to be positive for promoting cells to reach the inner cavities of the scaffold. The incorporation of trophic factors produced by hMSCs isolated from bone marrow is essential for cell adhesion and for final success of the PDMS-3D niche. In addition, the stiffness of Probe IV, used as scaffolding material, is very similar to cartilage stiffness and cartilage stands out for its large amount of extracellular matrix, mainly composed of collagen fibers.

In fact, the PDMS-3D niche, seeded with hMSCs and incubated in a chondrogenic medium during three weeks, realized chondrogenesis process expressing collagen type II validating a new method to obtain an excellent scaffold, at least, for cartilage and endochondral bone formation/repair strategies. Therefore, we believe that our scaffold's stiffness may have had a relevant impact on hMSCs growth and collagen generation, thus being potentially adequate for the promotion of chondrogenesis and for cartilage repair.

It is important to highlight that MSCs are adherent cells and rapidly die in suspension. Hence, mimicking the extracellular matrix is absolutely needed for prosthesis development and tissue repair biomaterials. However, cells do not usually get into a biomaterial unless using a pair antigen-antibody as hormone-receptor recognition system. To this end specific laser treatment is normally used to get reaction inside a biomaterial. Our novel approach shows a biomaterial where the communication factors promote MSC adhesion, even inside the pores. In addition, upon incubation in a regular chondrogenic medium, cells expressed collagen type II and most cells (whose nuclei are shown as bright red points in Figure 7) remained buried in collagen (shown in red in the images of Figure 7).

The design and manufacture process presented combines rapid prototyping, for controlling scaffold's outer geometry, with phase separation processes, for promoting porous biomimetic structures, towards final scaffolds and implants for tissue repair with desired physical properties and predefined geometries. Such process constitutes an alternative or a complementary approach to other recent solutions for the development of tissue engineering scaffolds, based exclusively on additive processes, which stand out due to the possibility of controlling final geometries from the design stage, although the CAD-based geometries are usually simple (typical wood-pile structures), soft, and not especially biomimetic. The detailed procedure can also complement or be combined with highly interesting nonintrusive $3 \mathrm{D}$ reconstructions based on medical imaging [45]. Future comparisons between niches directly obtained by additive manufacture from medical images and those obtained by casting with porogens in rapid molds will also provide additional information towards future applications.

Due to the results obtained, we recommend these scaffolds for cartilage and endochondral bone formation/repair strategies. We foresee relevant applications in several fields, such as biomedical and tissue engineering, that is, for the development of different (micro)implants and scaffolding structures, mainly for soft tissue replacement and repair. Personalized approaches are also possible, thanks to the combination of medical imaging and medical software resources with the capabilities of computer-aided design and additive rapid prototyping, what constitutes a relevant advantage in areas including aesthetic and reconstructive surgeries. 


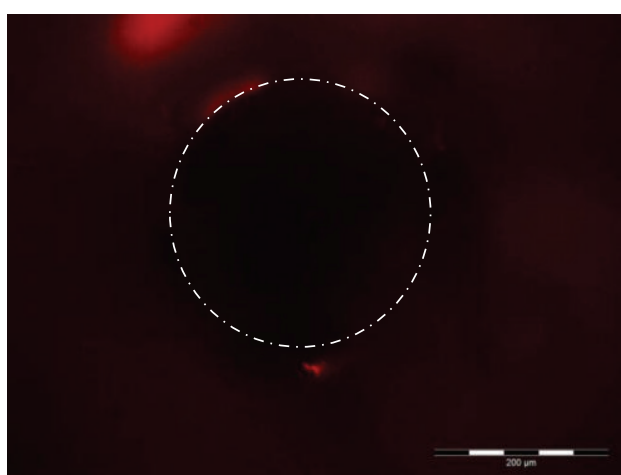

(a)

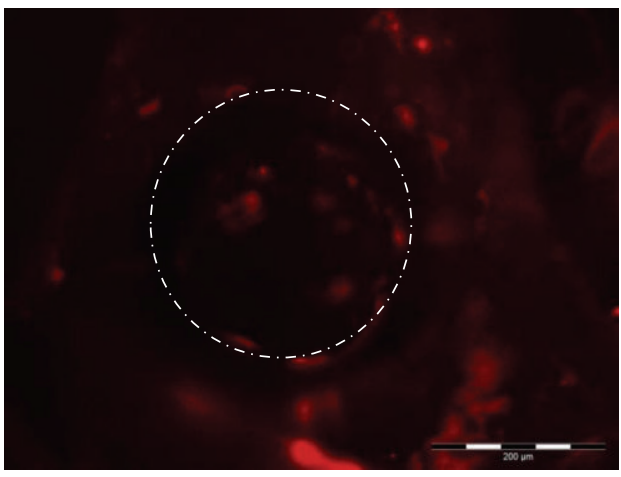

(c)

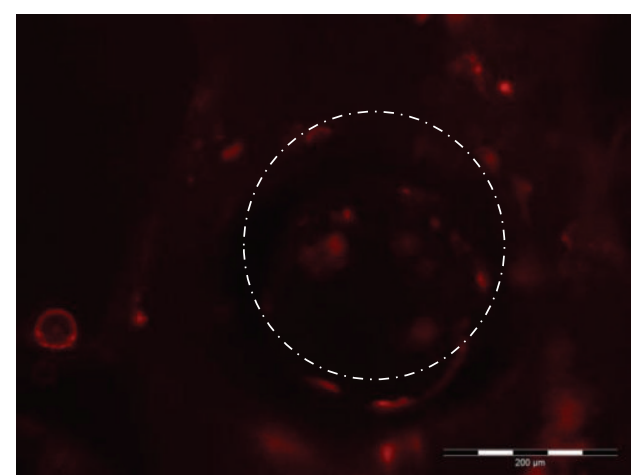

(b)

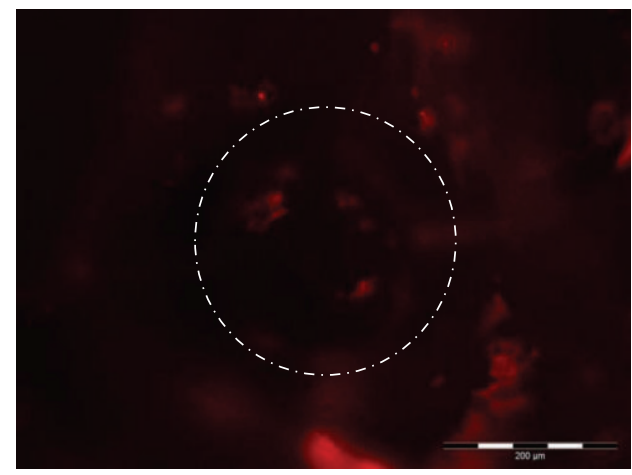

(d)

FIGURE 7: Results from hMSCs culture upon the PDMS sponges incorporating growth factors. Images taken at different depths to show cell penetration into pore (marked with dotted line). After a three-week culture process, cells (appreciated as prominent points in red) are well attached to the scaffold, have penetrated into the porous structure, and developed collagen (shown in red), helping to show the potential for tissue repair and cartilage generation of the proposed approach. Scale bar: $200 \mu \mathrm{m}$.

\section{Conclusions}

In this study we have presented a very promising approach for the rapid development of porous polydimethylsiloxane (PDMS) scaffold prototypes, with outer geometry defined from the design stage, according to the form of conventional implants or adapted to patients' biostructures. The manufacture method is based on phase separation processes using parts obtained by casting within additive rapid prototyped molds. We have included a comparative study of PDMS sponges obtained by different simple processes involving PDMS-sugar-water mixtures. By changing the proportions of these agents, main mechanical properties of the final scaffold can be tuned and adapted to the typical density and stiffness of several soft tissues, as has been previously detailed.

Different porous annuloplasty rings have been also obtained, by means of example, in order to highlight the potential of the manufacture process towards free-form porous implants and scaffolds for tissue replacement and repair. Final in vitro assessment was carried out using hMSCs (bone marrow-derived human mesenchymal stem cells), cultured onto porous PDMS scaffolds functionalized with APTS and equilibrated with trophic factors medium produced by the cells. Results show that porous PDMS scaffold prototypes are excellent 3D platforms for hMSCs adhesion. Furthermore, this PDMS-3D niche, seeded with
hMSCs and chondrogenic incubation medium during three weeks, showed a successful chondrogenesis determined by collagen type II expression. Thus, results show a versatile method to produce a $3 \mathrm{D}$ niche to further study questions about cartilage and endochondral bone formation or skeleton tissues clinical approaches.

\section{Conflict of Interests}

The authors declare that there is no conflict of interests regarding the publication of this paper.

\section{Acknowledgments}

This work was carried out with the support of the technologies available at the UPM Product Development Laboratory and counting with the help of Mr. Pedro Ortego for rapid prototyping tasks. The authors acknowledge reviewers for their positive opinions and proposals for improvement, which have helped to enhance paper quality and final result.

\section{References}

[1] R. Langer and J. P. Vacanti, “Tissue engineering," Science, vol. 260, no. 5110, pp. 920-926, 1993. 
[2] W. E. Thomas, D. E. Discher, and V. Prasad Shastri, "Mechanical regulation of cells by materials and tissues," MRS Bulletin, vol. 35, no. 8, pp. 578-583, 2010.

[3] W. L. K. Chen, M. Likhitpanichkul, A. Ho, and C. A. Simmons, "Integration of statistical modeling and high-content microscopy to systematically investigate cell-substrate interactions," Biomaterials, vol. 31, no. 9, pp. 2489-2497, 2010.

[4] A. Buxboim and D. E. Discher, "Stem cells feel the difference," Nature Methods, vol. 7, no. 9, pp. 695-697, 2010.

[5] A. Díaz Lantada, Handbook of Advanced Design and Manufacturing Technologies for Biodevices, Springer, 2013.

[6] P. J. S. Bartolo, H. Almeida, and T. Laoui, "Rapid prototyping and manufacturing for tissue engineering scaffolds," International Journal of Computer Applications in Technology, vol. 36, no. 1, pp. 1-9, 2009.

[7] J. Y. Tan, C. K. Chua, and K. F. Leong, "Indirect fabrication of gelatin scaffolds using rapid prototyping technology," Virtual and Physical Prototyping, vol. 5, no. 1, pp. 45-53, 2010.

[8] A. K. Ekaputra, Y. Zhou, S. M. Cool, and D. W. Hutmacher, "Composite electrospun scaffolds for engineering tubular bone grafts," Tissue Engineering A, vol. 15, no. 12, pp. 3779-3788, 2009.

[9] S. Lohfeld, M. A. Tyndyk, S. Cahill, N. Flaherty, V. Barron, and P. E. McHugh, "A method to fabricate small features on scaffolds for tissue engineering via selective laser sintering," Journal of Biomedical Science and Engineering, vol. 3, p. 138, 2010.

[10] R. Tzezana, E. Zussman, and S. Levenberg, "A layered ultraporous scaffold for tissue engineering, created via a hydrospinning method," Tissue Engineering C, vol. 14, no. 4, pp. 281-288, 2008.

[11] A. Díaz-Lantada, A. Mosquera, J. L. Endrino, and P. Lafont, "Design and rapid prototyping of DLC coated fractal surfaces for tissue engineering applications," Journal of Physics, vol. 252, no. 1, Article ID 012003, 2010.

[12] J. Stampfl, H. Fouad, S. Seidler et al., "Fabrication and moulding of cellular materials by rapid prototyping," International Journal of Materials and Product Technology, vol. 21, no. 4, pp. 285-296, 2004.

[13] R. Infuehr, N. Pucher, C. Heller et al., "Functional polymers by two-photon 3D lithography," Applied Surface Science, vol. 254, no. 4, pp. 836-840, 2007.

[14] P. S. Maher, R. P. Keatch, K. Donnelly, and J. Z. Paxton, "Formed 3D bio-scaffolds via rapid prototyping technology", in Proceedings of the 4th European Conference of the International Federation for Medical and Biological Engineering (ECIFMBE '08), vol. 22, pp. 2200-2204, November 2008.

[15] G. E. Ryan, A. S. Pandit, and D. P. Apatsidis, "Porous titanium scaffolds fabricated using a rapid prototyping and powder metallurgy technique," Biomaterials, vol. 29, no. 27, pp. 36253635, 2008.

[16] P. H. Warnke, T. Douglas, P. Wollny et al., "Rapid prototyping: porous titanium alloy scaffolds produced by selective laser melting for bone tissue engineering," Tissue Engineering C, vol. 15, no. 2, pp. 115-124, 2009.

[17] J. Stampfl, S. Baudis, C. Heller et al., "Photopolymers with tunable mechanical properties processed by laser-based highresolution stereolithography," Journal of Micromechanics and Microengineering, vol. 18, no. 12, Article ID 125014, 2008.

[18] I. Manjubala, A. Woesz, C. Pilz et al., "Biomimetic mineralorganic composite scaffolds with controlled internal architecture," Journal of Materials Science, vol. 16, no. 12, pp. 1111-1119, 2005.
[19] M. Schuster, C. Turecek, B. Kaiser, J. Stampfl, R. Liska, and F. Varga, "Evaluation of biocompatible photopolymers-I: photoreactivity and mechanical properties of reactive diluents," Journal of Macromolecular Science A, vol. 44, no. 5, pp. 547-557, 2007.

[20] M. Schuster, C. Turecek, A. Mateos, J. Stampfl, R. Liska, and F. Varga, "Evaluation of biocompatible photopolymers-II: further reactive diluents," Monatshefte fur Chemie, vol. 138, no. 4, pp. 261-268, 2007.

[21] F. Jung, C. Wischke, and A. Lendlein, "Degradable, multifunctional cardiovascular implants: challenges and hurdles," MRS Bulletin, vol. 35, no. 8, pp. 607-613, 2010.

[22] J. Y. Tan, C. K. Chua, and K. F. Leong, "Fabrication of channeled scaffolds with ordered array of micro-pores through microsphere leaching and indirect rapid prototyping technique," Biomedical Microdevices, vol. 15, p. 83, 2013.

[23] C. K. F. Chan, C.-C. Chen, C. A. Luppen et al., "Endochondral ossification is required for haematopoietic stem-cell niche formation," Nature, vol. 457, no. 7228, pp. 490-494, 2009.

[24] T. P. Richardson, M. C. Peters, A. B. Ennett, and D. J. Mooney, "Polymeric system for dual growth factor delivery," Nature Biotechnology, vol. 19, no. 11, pp. 1029-1034, 2001.

[25] A. Perets, Y. Baruch, F. Weisbuch, G. Shoshany, G. Neufeld, and S. Cohen, "Enhancing the vascularization of three-dimensional porous alginate scaffolds by incorporating controlled release basic fibroblast growth factor microspheres," Journal of Biomedical Materials Research A, vol. 65, no. 4, pp. 489-497, 2003.

[26] M. W. Laschke, M. Rücker, G. Jensen et al., "Incorporation of growth factor containing Matrigel promotes vascularization of porous PLGA scaffolds," Journal of Biomedical Materials Research A, vol. 85, no. 2, pp. 397-407, 2008.

[27] J. Chen, R. Zjang, and W. Wang, Fabricating Microporous PDMS Using Water-in-PDMS Emulsion, RSC Publishing Blogs Home. Chips and Tips, 2012.

[28] S. Peng, P.G. Hartley, T.C. Hughes, and Q. Guo, "Controlling morphology and porosity of porous siloxane membranes through water content of precursor microemulsion," Soft Maters, vol. 8, no. 40, p. 10493, 2012.

[29] P. K. Yuen, H. Su, V. N. Goral, and K. A. Fink, "Threedimensional interconnected microporous poly(dimethylsiloxane) microfluidic devices," Lab on a Chip, vol. 11, no. 8, pp. 1541$1544,2011$.

[30] Q. Tan, S. Li, J. Ren, and C. Chen, "Fabrication of porous scaffolds with a controllable microstructure and mechanical properties by porogen fusion technique," International Journal of Molecular Sciences, vol. 12, no. 2, pp. 890-904, 2011.

[31] F. Ciaramella, V. Jousseaume, S. Maitrejean, B. Rémiat, M. Verdier, and G. Passemard, "Mechanical properties of porous MSQ films: impact of the porogen loading and matrix crosslinking," in Proceedings of the Materials Research Society Spring Meeting, pp. 17-22, April 2005.

[32] W. Y. Yeong, N. Sudarmadji, H. Y. Yu et al., "Porous polycaprolactone scaffold for cardiac tissue engineering fabricated by selective laser sintering," Acta Biomaterialia, vol. 6, no. 6, pp. 2028-2034, 2010.

[33] A. Díaz Lantada, R. D. Valle-Fernández, P. L. Morgado et al., "Development of personalized annuloplasty rings: combination of CT images and CAD-CAM tools," Annals of Biomedical Engineering, vol. 38, no. 2, pp. 280-290, 2010.

[34] M. F. Pittenger, A. M. Mackay, S. C. Beck et al., "Multilineage potential of adult human mesenchymal stem cells," Science, vol. 284, no. 5411, pp. 143-147, 1999. 
[35] D. P. Lennon, S. E. Haynesworth, S. P. Bruder, N. Jaiswal, and A. I. Caplan, "Human and animal mesenchymal progenitor cells from bone marrow: identification of serum for optimal selection and proliferation," In Vitro Cellular and Developmental Biology, vol. 32, no. 10, pp. 602-611, 1996.

[36] S. Ogueta, J. Muñoz, E. Obregon, E. Delgado-Baeza, and J. P. García-Ruiz, "Prolactin is a component of the human synovial liquid and modulates the growth and chondrogenic differentiation of bone marrow-derived mesenchymal stem cells," Molecular and Cellular Endocrinology, vol. 190, no. 1-2, pp. 51-63, 2002.

[37] M. Romero-Prado, C. Blázquez, C. Rodríguez-Navas et al., "Functional characterization of human mesenchymal stern cells that maintain osteochondral fates," Journal of Cellular Biochemistry, vol. 98, no. 6, pp. 1457-1470, 2006.

[38] A. Javed, B. Guo, S. Hiebert et al., "Groucho/TLE/R-esp proteins associate with the nuclear matrix and repress RUNX $(\mathrm{CBF} \alpha / \mathrm{AML} / \mathrm{PEBP} 2 \alpha)$ dependent activation of tissue-specific gene transcription," Journal of Cell Science, vol. 113, no. 12, pp. 2221-2231, 2000.

[39] J. Normatov and M. S. Silverstein, "Porous interpenetrating network hybrids synthesized within high internal phase emulsions," Polymer, vol. 48, no. 22, pp. 6648-6655, 2007.

[40] J. Y. Wong and J.D. Bronzino, Biomaterials, CRC-Press, 2007.

[41] F. J. Rojo, J.M. Atienza, E. Jorge-Herrero, J.M. García-Páez, and G.V. Guinea, "Resistencia a tracción de membranas de pericardio para válvulas cardiacas biológicas," Anales de Mecánica de la Fractura, vol. 26, no. 1, 2009.

[42] R. K. Goyal, P. Biancani, A. Phillips, and H.M. Spiro, Mechanical Properties of the Esophageal Wall, Yale University, 1971.

[43] I. Levental, P. C. Georges, and P. A. Janmey, "Soft biological materials and their impact on cell function," Soft Matter, vol. 3, no. 3, pp. 299-306, 2007.

[44] J. M. Mansour, "Biomechanics of cartilage," in Kinesiology: The Mechanics and Pathomechanics of Human Movement, C. A. Oatis, Ed., Lippincott Williams and Wilkins, 2003.

[45] A. Tsouknidas, A. Lontos, S. Savvakis, and N. Michailidis, "Nonintrusive 3D reconstruction of human bone models to simulate the biomechanical response," 3D Research Express, vol. 3, no. 2, p. 5, 2012. 

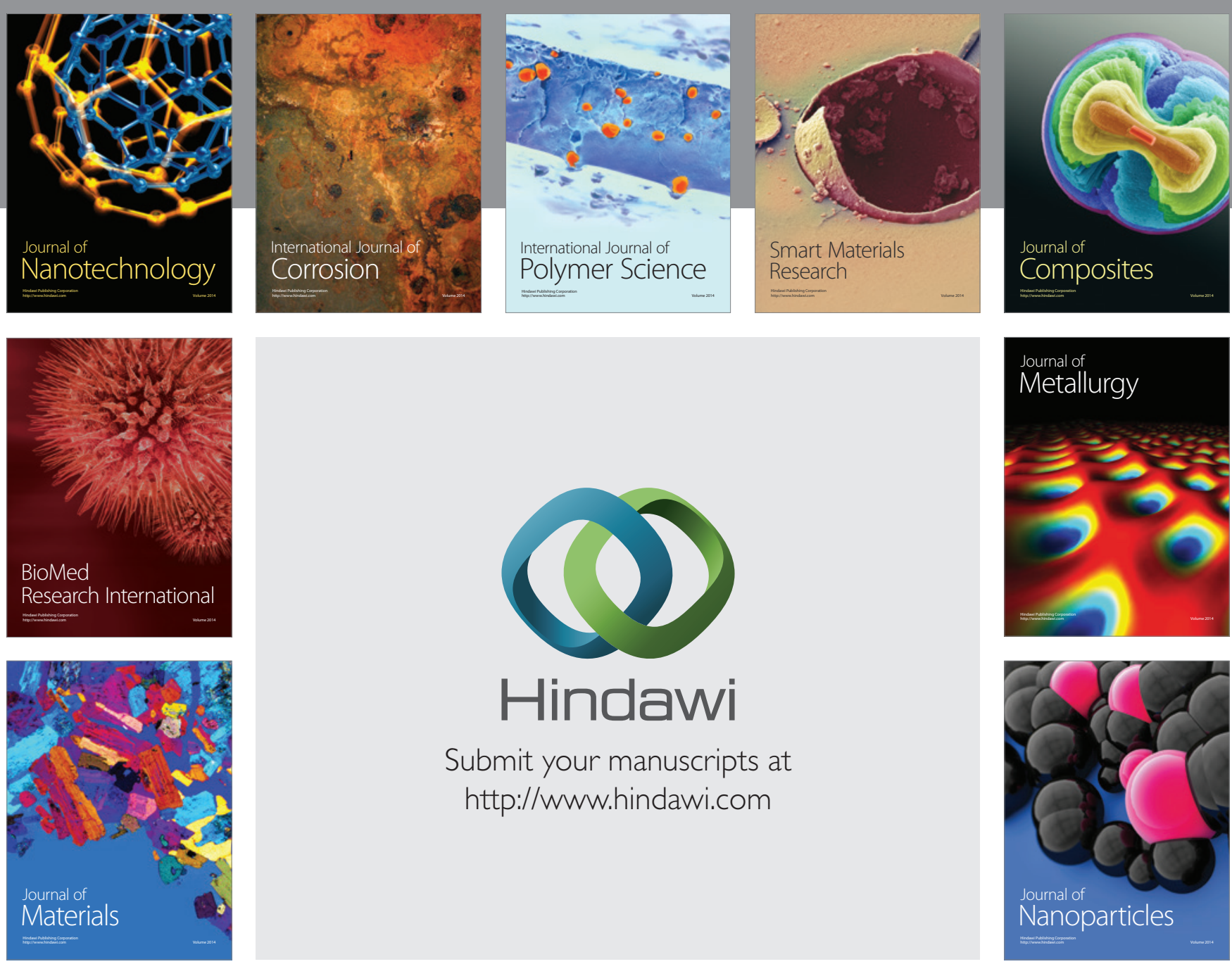

Submit your manuscripts at http://www.hindawi.com
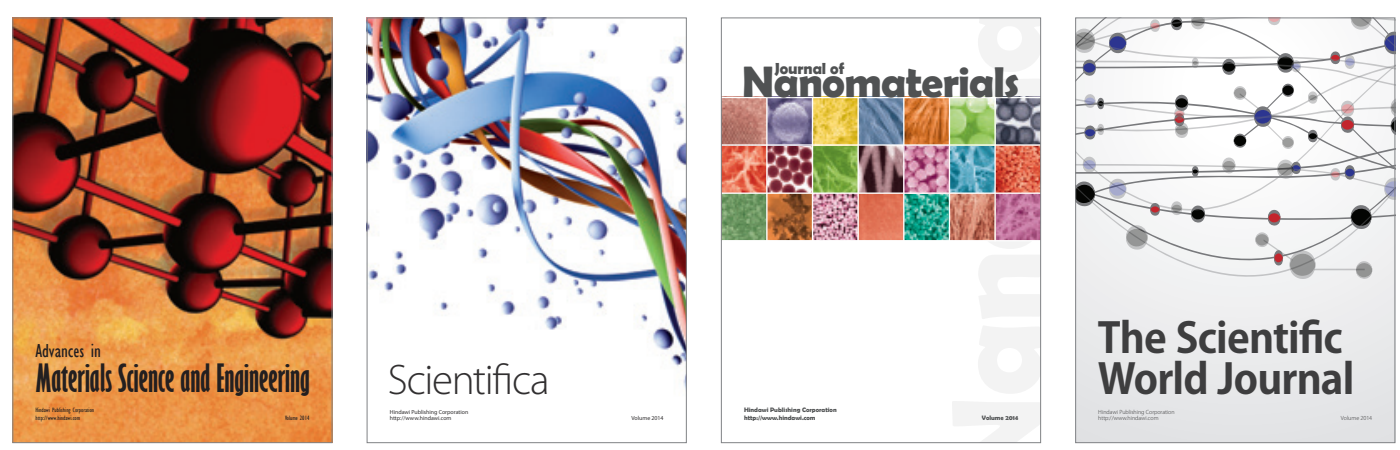

\section{The Scientific World Journal}
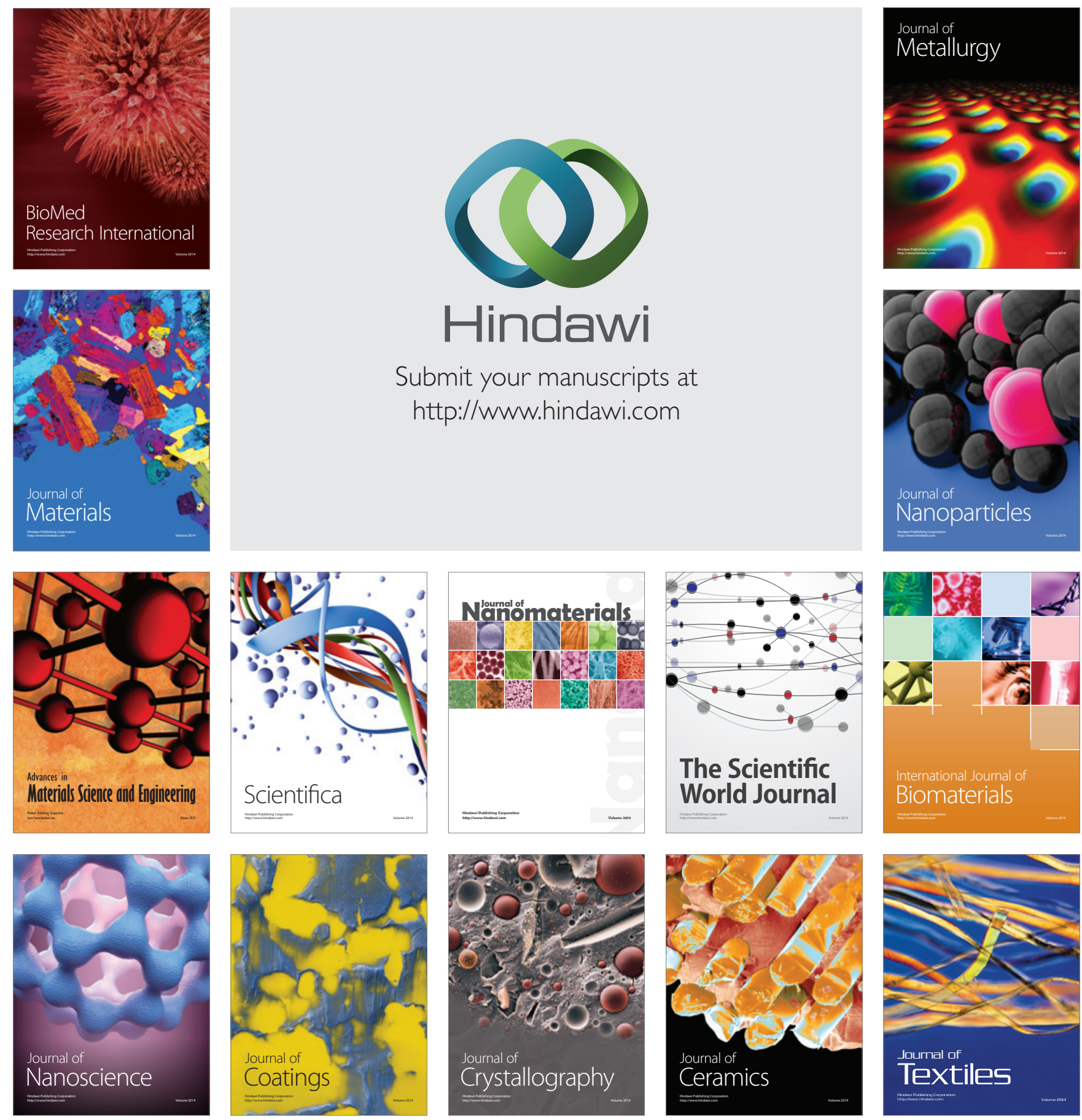